\title{
LA EDUCACIÓN PARA LA SALUd COMO OBJETO DE ESTUDIO HISTÓRICO: PROYECTO DE INVESTIGACIÓN.
}

\section{THE HEALTH EDUCATION AS AN OBJECT OF HISTORICAL STUDY: RESEARCH PROYECT}

SALVADOR SÁEZ ${ }^{a}$

MIGUELA MARTÍNEZ $a, b$

JUAN TORRES ${ }^{a, c}$

CARMEN TORRES PENELLA a

LORENA LOURDES TEJERO VIDAL ${ }^{\mathrm{a}, \mathrm{e}}$ ROSER PALAU COSTAFREDA ${ }^{f}$
a: Departament d'Infermeria. Universitat de Lleida.
b: Centre d'Atenció Primària Balàfia. Institut Català de la Salut
c: Serveis Regionals a Lleida de I'Agència de Salut Pública de Catalunya.
e: Hospital Santa Maria. Gestió de Serveis Sanitaris. Lleida.
f: Hospital Universitari Mútua de Terrassa.

E-mail contacto: carme.torres@infermeria.udl.cat 


\section{RESUMEN}

\section{Título:}

La Educación para la Salud como objeto de estudio histórico: Proyecto de investigación.

\section{Objetivo:}

El objetivo es presentar el proyecto de investigación cuyo propósito es conocer la evolución de la Educación para la salud (EpS) desde la antigüedad hasta la época contemporánea, con el fin de identificar y comprender la génesis y construcción de la disciplina.

\section{Método:}

Se trata de un estudio cualitativo histórico enmarcado en el paradigma interpretativo etnohistórico y hermenéutico. Los pilares básicos en que se enmarca conceptualmente, son la Educación, la Persona, grupo o comunidad y la Salud. El espacio social, es aquel en que los grupos sociales vivían y el estructural-temporal, las épocas históricas: prehistoria, antigüedad (culturas antiguas y clásicas), edad media, renacimiento y contemporánea hasta pasada la Guerra Civil española (1940).

El sujeto de estudio es la EpS a partir de los elementos que la conforman: el concepto de salud, las creencias, los conocimientos sanitarios, las intervenciones y los recursos educativos para la salud de les personas y los grupos sociales en cada una de las épocas históricas a estudio.

Las fuentes utilizadas son las indirectas, materiales-arqueológicas y culturales: verbales (escritas) y no verbales (semiológicas/audiovisuales) y las no seriadas. La recogida de información a través de técnicas de investigación histórica cualitativa: la observación y análisis documental bibliográfico, iconográfico de archivos, prensa, publicaciones oficiales, textos bibliográficos y técnicas textuales-filosóficas: análisis de contenido y crítica histórica.

El análisis de la información será cronológico y mediante una clasificación por temáticas y periodos históricos.

Palabras clave: Educación para la salud. Historia. Proyecto de investigación. 


\section{ABSTRACT}

\section{Title:}

The history of Health Education, a subject under study. Research project.

\section{Objetive:}

The aim is to present a research project, which starts with the evolution of Health Education (HE) through the ages to arrive at an understanding of the origin and construction of the discipline.

\section{Method:}

This is done through a qualitative historic study framed in the interpretative paradigm of ethnohistory and hermeneutics. The organising concepts of this paradigm are: the education; the person; the group or community; the historical eras: the pre-history; the antiquity (ancient and classical cultures); the Middle Ages; the Renaissance and the Recent times up to the end of the Spanish Civil War.

The subject under study is Health Education beginning from its constituent parts (the concept of health; beliefs; knowledge of hygiene; interventions and educational resources for personal and group health) in each of the historical eras under study.

Sources are indirect - archaeological, cultural, verbal both written and semiotic/audiovisual and those not serialies. Gathering of information through the techniques of qualitative history: observation and analysis of written material, visual art, newspapers, official publications, bibliographies and textual-philosphic techniques (analysis of primary and secondary historical sources).

The analysis of information will be chronological and by subject classification and by historical periods.

\section{Key words:}

Health Education, history, research. 


\section{INTRODUCCIÓN.}

La Educación para la Salud (EpS) como disciplina ha experimentado un gran desarrollo desde la mitad del siglo XX hasta nuestros días, en ella han contribuido las teorías sobre la salud, la educación y las necesidades de las personas, grupos o comunidad. Sin embargo su practica creemos ha estado presente desde el inicio de la vida humana ligada al instinto de supervivencia.

El objetivo de este artículo es presentar el diseño de proyecto de investigación cuyo propósito es conocer la evolución de la EpS desde la antigüedad hasta la época contemporánea con el fin de identificar y comprender la génesis y construcción de la disciplina.

\subsection{Antecedentes y estado de la cuestión.}

Los estudios existentes sobre vestigios o rastros históricos de la EpS son escasos, no obstante, los más relacionados con ella tienen que ver principalmente con la salud pública. Salleras ${ }^{1}$ en su obra Educación sanitaria, hace una introducción histórica de las relaciones existentes entre grupos humanos de las civilizaciones egipcia, hebrea, griega, e imperio romano, y las prácticas relacionadas con la higiene personal y de salud pública, concluyendo que el despertar de la conciencia sanitaria no se produjo hasta el inicio de la edad contemporánea.

De civilizaciones antiguas, Pardo ${ }^{2}$ destaca la contribución del judaísmo en la salud pública, exponiendo los conocimientos de la misma, prácticas medicosanitarias, higiene alimentaría, medidas preventivas de contagio, higiene sexual y las normas religioso-sanitarias y salud del pueblo judío.

Valadez ${ }^{3}$, nos dice también que la EpS es una práctica antigua que ha 
variado en cuanto a su enfoque ideológico y metodológico como consecuencia de los cambios paradigmáticos en relación al concepto de salud dominante y la manera de ejercer la práctica educativa y de salud.

De la Guerra Civil española, Corbella ${ }^{4}$ apunta el importante papel que tuvieron los carteles de propaganda sanitaria destinados principalmente a disponer de hombres con óptimas condiciones físicas para la lucha.

Pelicioni ${ }^{5}$, hace una retrospectiva histórica de acciones educativas en el campo de la salud, especialmente la educación sanitaria y la educación en salud pública, así como sus relaciones con la promoción de la salud. Concluye que el empoderamiento y la participación activa de la gente se harán posibles por la educación para la salud como proceso político de formación para la ciudadanía preparando a individuos y/o a grupos para tomar el control y la responsabilidad por su propia salud.

La literatura científica del pasado de la EpS es escasa, o lo que es lo mismo, una historia de la EpS resulta difícil de vertebrar. ¿Acaso la educación para la salud es una práctica contemporánea o ha existido siempre? ¿Se educaba en salud en épocas pasadas? ¿Cuáles han sido las finalidades últimas de la EpS a lo largo de la historia?

\subsection{Justificación.}

Como formadores universitarios en EpS de grado y postgrado, al querer escrutar las fuentes de la EpS, hemos detectado cierta carencia bibliográfica de recorrido histórico de la misma anterior al siglo XX. Por este motivo creemos pertinente teóricamente, socialmente, profesionalmente y disciplinariamente investigar el pasado sobre los conocimientos y prácticas de EpS que llevaban a cabo las personas y grupos sociales en las distintas épocas y culturas históricas. 


\section{OBJETIVOS.}

Objetivo general: Conocer y analizar la evolución de la EpS desde la antigüedad hasta el siglo XX contribuyendo así a identificar y comprender la génesis y construcción de la disciplina.

El propósito interno es que pueda servir de plataforma para canalizar las inquietudes en forma de trabajo fin de master, tesis doctorales etc. del alumnado de grado y posgrado sobre la EpS. Recopilar las aportaciones en soportes multimedia para su uso en la enseñanza-aprendizaje de la Historia de la EpS. Finalmente elaborar un manual sobre la Historia de la Educación para la Salud. Para ello se prevé buscar financiación para llevar a cabo el proyecto.

\section{PLAN DE ESTUDIO.}

\subsection{Tipo de estudio.}

Se trata de un estudio cualitativo histórico enmarcado en el paradigma interpretativo etnohistórico y hermenéutico ${ }^{6}$. Etnohistórico porque implica el recuento de la realidad actual de la EpS como producto de sucesos históricoculturales a partir de la exploración y descripción del significado de los elementos que caracterizan la EpS en una determinada época o cultura histórica. Hermenéutica por que requiere un trabajo de interpretación y análisis de comprensión del significado de textos, objetos y conductas humanas del pasado relacionadas con la EpS.

El marco conceptual o pilares básicos en que reposa o se enmarca conceptualmente la EpS, son la Educación, la Persona, grupo o comunidad y la Salud.

\subsubsection{La Educación.}

De las diferentes corrientes en educación se propone como referente la 
enseñanza aprendizaje de aproximación humanista. El término Educación humanista $^{7}$ es generalmente empleado para designar diversas teorías y prácticas comprometidas con la visión del mundo y con el código de ética del humanismo; o sea, la propuesta de intensificación del desarrollo, del bienestar y de la dignidad como objetivos últimos de todo pensamiento y acción humanas, por encima de los ideales y valores religiosos, ideológicos o nacionales. Más allá de eso, con base en una larga tradición filosófica y moral, que va desde los antiguos profetas bíblicos y filósofos griegos hasta la Declaración Universal de los Derechos Humanos y la declaración Universal de los Derechos de los Niños de la ONU.

Durante el Renacimiento es cuando nace la idea de la educación como una función de la vida. Más tarde en el siglo XVIII, Jean Jaques Rousseau (1712-1780) describía en sus escritos una concepción de la educación basada en la libertad de aprender. Para él, el aprendiz no tan solo era el objeto de la educación, sino que era la fuente. Ya más cerca de nosotros Montesouri (1952) insiste sobre la educación basada en las potencialidades, las necesidades y los intereses de niños y adultos. Desde el año 1940 Carl Rogers había desarrollado una concepción de la educación basada en el aprendizaje significativo o de la experiencia. Todos estos pedagogos se adhieren al concepto de psicología humanista y ven al aprendiz como individuo capaz de experimentar un crecimiento psicológico óptimo para el desarrollo libre de la plenitud de sus posibilidades. En una palabra, la persona que aprende es creador en continuo cambio ${ }^{8}$.

\subsubsection{La Persona.}

Conjuntamente al concepto de educación del ser humano de orientación humanista, de cara a identificar los recursos y las intervenciones educativas en salud de la persona y/o grupos, creemos necesario fundamentar la concepción de persona. Por su carácter universal, se toma la visión integral que de la 
persona nos ofrece la filosofía en cuidados de enfermería de Henderson ${ }^{9}$, como un todo complejo compuesto de catorce necesidades básicas o fundamentales, clasificadas a la vez en bio-fisiológicas: respirar; comer y beber; eliminar por todas las vías; mantener la temperatura del cuerpo; vestir y desvestir; mover y mantener posturas correctas; dormir y descansar; mantener la higiene y cuidado de la piel y anejos; y las sociales: evitar peligros; comunicar; vivir según creencias; sentirse útil; recrearse, y aprender.

La necesidad de aprender de Henderson puede considerarse como la necesidad maestra en EpS. Esta requiere de los profesionales de la salud, ofrezcan a la comunidad su ayuda en el aprendizaje de los conocimientos, las actitudes y habilidades saludables inherentes al conjunto de todas las necesidades humanas y motivar que realicen unos autocuidados en salud adecuados para que puedan desarrollar el máximo potencial de satisfacción de cada necesidad.

Así pues, como centro de las intervenciones de EpS se adopta el marco de referencia de las necesidades de Henderson, tomando como necesidad maestra la necesidad de aprender en clara sintonía conceptual con la EpS.

\subsubsection{La Salud.}

Desde la muy conocida concepción que dio de la salud la Organización Mundial de la Salud (OMS) en el 1947, de "estado de completo bienestar físico mental o social", han sido muchos los autores que con distintas perspectivas han definido la salud. Escogemos la de Kickbush ${ }^{11}$ por su carácter cotidiano "La salud es básicamente una estructura social: se crea a través de la interrelación entre la gente y sus entornos en el proceso de la vida diaria: donde la gente vive, ama, aprende, trabaja y juega."

Los aspectos relacionados con la salud de la comunidad y la salud pública 
están hoy en nuestro país regulados por ley. En el artículo 2 de la "Llei 18/2009 del Parlament de Catalunya"12 define la Promoción de la Salud como el conjunto de actuaciones, prestaciones y servicios destinados a fomentar la salud individual y colectiva y a impulsar la adopción de estilos de vida saludables mediante intervenciones adecuadas en materia de información, comunicación y educación sanitaria. En el mismo artículo, no existe una definición específica de Educación para la salud, sino que ésta la contempla como estrategia o elemento de promoción de la salud en forma de intervención educativa en salud.

La EpS como término genérico se ha utilizado para describir cualquier esfuerzo de proporcionar información y conocimientos relativos al mantenimiento y promoción de la salud, cosa que implica que incluya aspectos formales e informales. Históricamente la EpS ha pasado por diferentes etapas según el contexto en el que se ha desarrollado desde hace siglos hasta la actualidad. Salleras ${ }^{13}$ distingue dos grandes etapas o periodos en la evolución del concepto y contenido de la educación sanitaria. El primer periodo o "clásico" que va des de el principio del siglo XX, el concepto de educación sanitaria solo incluía las acciones educativas dirigidas al individuo y que tendían a responsabilizarle de su propia salud, y su objetivo único eran las conductas relacionadas con el fomento y la protección de la salud. Se trataba, pues de modificar las conductas insanas relacionadas con lo que hoy en día se conoce como promoción de la salud, mediante actividades educativas o persuasivas dirigidas al individuo, al que responsabilizaba de su propia salud.

La segunda etapa ya a partir de los 70, considerada como etapa "actual" los conceptos y objetivos sufrieron un cambio. Desde los años 60 numerosas investigaciones ya habían demostrado que las acciones de educación sanitaria dirigidas solo al individuo habían sido poco eficaces en la modificación de los comportamientos insanos frecuentemente enraizados en la sociedad. Por todo 
ello, la modificación de los factores externos en sentido favorable, paso a ser uno de los objetivos de la educación sanitaria, la cual además de incidir sobre los individuos, debe promover también cambios ambientales y sociales que sean necesarios para que el cambio de conducta pueda llevarse a cabo y mantenerse. Además, la educación de pacientes pasó a formar parte de la educación sanitaria, al comprobar que la conducta humana, no solo es importante en la fase de promoción de la salud, sino que también lo es en la restauración de la salud, sobretodo de las enfermedades crónicas.

Según el glosario de Nutbeam ${ }^{14}$ la EpS comprende las oportunidades de aprendizaje creadas conscientemente que suponen una forma de comunicación destinada a mejorar la alfabetización sanitaria, incluye la mejora del conocimiento de la población en relación con salud y el desarrollo de habilidades personales que conducen a la salud individual y de la comunidad.

La EpS es considerada hoy como una estrategia más de promoción de la salud.

\section{2. Ámbito de estudio.}

La localización o el espacio social e histórico, son aquellos en el que los grupos sociales vivían. Inicialmente, en una fase exploratoria será universal, sin descartar acotarlo en función del estudio. El espacio estructural-temporal, las épocas históricas: Prehistoria, Antigüedad (culturas antiguas y clásicas), Edad Media, Renacimiento y Contemporánea hasta pasada la Guerra Civil española (1940).

\subsection{Sujeto de estudio.}

La EpS a partir de los elementos o características que la conforman: el concepto de salud, las creencias, los conocimientos sanitarios, las actividades/intervenciones y los recursos educativos para la salud de las 
personas y los grupos sociales en cada una de las épocas históricas a estudio.

\subsection{Fuentes.}

Se denomina fuente histórica a todo aquel objeto material, instrumento o herramienta, símbolo o discurso intelectual, que procede de la creatividad humana, a través del cual puede inferirse algo acerca de una determinada situación social en el tiempo Los tipos de fuentes que se emplearan para este estudio son las indirectas, materiales-arqueológicas y culturales: verbales (escritas) y no verbales (semiológicas/audiovisuales) y las no seriadas ${ }^{15}$.

Las fuentes etnográficas para la historia de la enfermería y por extensión la de una de sus funciones, la educadora, son todas aquellas que contienen referencias sobre las creencias, los comportamientos y las situaciones en las que se han dado los cuidados de salud en los diferentes contextos históricos siendo, por su naturaleza, especialmente relevantes y pertinentes para vertebrar también una historia de la EpS en la que tienen cabida los documentos escritos, orales e iconográficos ${ }^{16}$.

La información se extraerá de documentos, bibliografía, iconografía, etc. Las bases de datos consultadas hasta la fecha han sido: Cuiden, CINAHL, Cuidatge, ProQuest, Medline.

\subsection{Recogida y análisis de la Información.}

Como técnicas de investigación histórica cualitativa se utilizará la observación documental: lectura, observación y análisis documental, bibliográfico, iconográfico de archivos, prensa, publicaciones oficiales, textos bibliográficos y técnicas textuales-filosóficas: análisis de contenido y crítica histórica ${ }^{17}$. Todas ellas en función a la adecuación del estudio de investigación. 
La información extraída de los documentos, bibliografía, iconografía etc., se observará y analizará en función a la adecuación del estudio, es decir, si el documento es capaz de responder al mayor número de preguntas de investigación planteadas, con menos problemas de fiabilidad, menos posibilidad de equivocarse o mejor adaptación a la finalidad de la investigación.

El análisis de la información se llevará a cabo de forma cronológica y mediante una clasificación por temáticas y periodos históricos.

El registro de la información se ayudará de una parrilla elaborada a tal efecto.

\subsection{Aspectos éticos.}

A lo largo de todo el estudio se seguirá las normativas de reproducción y divulgación documental y de otras fuentes establecida por cada uno de los archivos, o entes y solicitando las autorizaciones oportunas.

\section{BIBLIOGRAFÍA.}

1) Salleras L. Educación sanitaria. Principios, métodos, aplicaciones. Madrid: Diaz de Santos, S.A.;1990; 31-48.

2) Pardo, C. Judaísmo y salud. Revista Enfermería Científica. 1992; 129: 4-7.

3) Valadez I; Villaseñor M; Alfa N. Educación para la salud, la importancia del concepto. Revista de Educación y desarrollo. 2004; 1: 43-8.

4) Corbella, J. Notes sobre cartellisme sanitari de la Guerra Civil. Gimbernat: revista catalana d'història de la medicina i de la ciència [en línia], 1998;30:129-40.

http://84.88.10.30/index.php/Gimbernat/article/view/44705 [Consultado: 16-04-12]. 
5) Pelicioni, M C; Pelicioni, A. Educação e promoção da saúde: uma retrospectiva histórica. O Mundo da Saúde. 2007; 31(3):320-28.

6) Álvarez-Gayou JL. Cómo hacer investigación cualitativa. Fundamentos y metodología. Barcelona: Paidós Ibérica; 2005. p.65-99.

7) Aloni, N. Hakibbutzim College of Educatión-Israel. http://arje.hotusa.org/filoedu1.htm. consultado el 25-04.2012.

8) Riopelle L; Grondin L; Phaneuf M. Soins infirmiers. Enseignement à la clientèle. Quebec: MacGraw-Hill; 1988. p. 3-26.

9) Henderson V. Principios básicos de los cuidados de enfermería. Ginebra: Consejo Internacional de Enfermería;1971.

10) Delchambre N; Lefevre MR; Ligot A; Mainjot N; Marlière MF. Guide d'observation des 14 besoins de l'être humain; $2^{\mathrm{e}}$ édition. Bruxelles: De Boeck Université; 2000.

11) Kickbusch I, Promoción de la salud a través de La Educación de Adultos. En: Quinta conferencia Internacional de Educación de las Persona Adultas. Hamburgo, CONFITEA;1997. p.6.

12) Parlament de Catalunya. Llei, 18/2009, del 22 d'octubre de salut pública. de salut pública. Barcelona: Publicacions del Parlament de Catalunya; 2009.p.23-5.

13) Salleras L. Educación sanitaria. Principios, métodos, aplicaciones. Madrid: Diaz de Santos S.A.;1990; 52-64.

14) Nutbeam D. Promoción de la Salud. Glosario. O.M.S. 1998. p. 13. http://www.bvs.org.ar/pdf/glosario sp.pdf.Consultado 14 de mayo de 2012.

15) Aróstegui, J. La investigación historica. Barcelona: Crítica: 2001. p. 379-88.

16) Siles J. Fuentes etnográficas e historia de la enfermería. Híades. Revista de Historia de la Enfermería, no 10. Qalat Chábir, A.C. Alcalá de Guadaíra (Sevilla) 2008; 10:111-37.

17) Siles J. Historia de la Enfermería. Alicante: Aguaclara; 1999. p. 16-87. 